\title{
The Structure and Coherence of the New Environmental Paradigm: Reconceptualizing the Dimensionality Debate
}

\author{
Chenyang Xiao' \\ Department of Sociology \\ American University, Washington, DC, United States \\ Jan Buhrmann \\ Department of Sociology \\ Illinois College, Illinois, United States
}

\section{Abstract}

This research examines the structure and coherence of an emerging environmental world view as measured by the new environmental paradigm (NEP) scale, developed by Dunlap and Van Liere (1978). We utilize data from two independent surveys of three communities in Colorado and Wyoming, United States, in 1997 and 2012, and examine the extent to which this world view has been accepted by the general public. Using factor analysis, we focus our attention on the structure and coherence of the NEP, as well as the dimensionality of this scale, revisiting the original question raised by Dunlap and Van Liere of whether a coherent ecological world view has emerged among the general public at this point in time. Comparisons between the two surveys reveal that both the structure and coherence of the NEP are highly consistent and stable over time, lending support to the belief that the general public within our study areas has indeed developed such a world view.

Keywords: belief constraints, belief systems, environmental beliefs, environmental world view, new ecological paradigm, new environmental paradigm

\section{Introduction}

In the midst of heightened public concern for environmental issues from the late 1960s through the 1970s, Dunlap and colleagues among several social researchers believed there might be a paradigmatic shift in society, a new ecologically oriented world view named the new environmental paradigm (NEP), emerging to challenge

1 Corresponding author: xiao@american.edu 
the anti-ecological dominant social paradigm (DSP) (see Dunlap, 2008 for a review). The DSP reflects the prevailing values and beliefs that favor a free-market economy, advancements in science and technology, as well as never-ending growth and progress, which were argued by many as one major cause of more and more salient environmental problems. The NEP, in contrast, represents such beliefs that there is an inevitable limit to economic growth, and that humans are not above nature, but instead depend on an ecologically balanced nature to survive (e.g., Dunlap \& Van Liere, 1978; Pirages \& Ehrlich, 1974). To capture this change in the world view regarding human-environment relations, Dunlap and Van Liere (1978) created an instrument, the NEP scale, which was updated to the new ecological paradigm (still NEP) scale in 2000. Development of the updated scale was for the purpose of uncovering a wider range of factors that potentially comprise an ecological world view, offering a better balance of pro- and anti-environmental items, and updating the terminology of the specific items (Dunlap et al., 2000). The NEP scale has since become the most widely used measure of environmental concern worldwide (Hawcroft \& Milfont, 2010).

Naturally, such a widely used instrument has received a great deal of criticism regarding its measurement quality (Amburgey \& Thoman, 2012; Dunlap et al., 2000; Hawcroft \& Milfont, 2010). One of the most persist criticisms is that the NEP scale is not unidimensional (Dunlap, 2008). Almost immediately after the creation of the original NEP scale, Albrecht et al. (1982) found that although the scale appears to have adequate internal consistency and validity, it seems to have three dimensions that are only mildly interrelated. This tripart factor structure was then somewhat confirmed by Geller and Lasley (1985). Since that time, a number of studies have found that the NEP scale, in both the original and the revised versions, has two or more dimensions (e.g., Bechtel et al., 2006; Corral-Verdugo \& Armendariz, 2000; Edgell \& Nowell, 1989; Englis \& Phillips, 2013; Fleury-Bahi et al., 2015; Furman, 1998; Gooch, 1995; Noe \& Snow, 1990; Schuett \& Ostergren, 2003; Woodworth et al., 2011).

It is noticeable, however, that there seems to be little consensus on the factor structure of the NEP scale, in terms of both the number of factors and which individual items load on which factors (Hawcroft \& Milfont, 2010). A significant amount of disagreement in the empirical findings has led Dunlap et al. (2000) to argue that most findings are likely sample-specific, and therefore there is not yet decisive enough evidence to draw conclusions about the multidimensionality of the NEP scale. We believe the current empirical literature on the dimensionality of the NEP scale is rather difficult to make sense of, given the lack of a consensus on the factor structure of the NEP.

We believe a more productive alternative is to return to the original question when the scale was created almost four decades ago. While it appears that academics and intellectuals endorse the world view as measured by the NEP scale, to what 
extent has such a world view been accepted by the general public (Dunlap \& Van Liere, 1978)? By raising this question, we argue it is time to reconceptualize the dimensionality debate as more than a methodological issue, even though the methodological concern remains important in this debate. Instead, we should pay attention to a more conceptual question: what is the status of the supposed paradigmatic shift from the DSP to the NEP for the general public? In this paper we first lay out the reasons why we believe this alternative approach will be more productive. Then we reexamine the factor structure of the original NEP scale using data from identical surveys conducted at two different points in time to demonstrate there is a significant amount of evidence for an ecological world view with regard to the general public.

\section{The dimensionality debate}

Much of the attention on the dimensionality of the NEP scale is methodological in nature. As in the case of any multi-item scale, it is critical to make sure that all items measure the same underlying construct, which necessitates examination of a scale's internal consistency and dimensionality. Up to this point, we can see studies demonstrating that the 12-15 items in the original and revised NEP scales load onto two (e.g., Gooch, 1995; Ji, 2004; Noe \& Snow, 1990; Nooney et al., 2003; Van Riper \& Kyle, 2014), three (e.g., Albrecht et al., 1982; Edgell \& Nowell, 1989; Englis \& Phillips, 2013; Geller \& Lasley, 1985), four (e.g., Erdogan, 2013; Furman, 1998; Khan et al., 2012), and even five or more underlying factors (e.g., Brennan et al., 2014; Woodworth et al., 2011; Wu, 2012). Furthermore, while some studies find factors corresponding to the hypothesized facets as identified in Dunlap and Van Liere (1978) and Dunlap et al. (2000) (e.g., Albrecht et al., 1982; Amburgey $\&$ Thoman, 2012; Woodworth et al., 2011), most studies do not.

Aside from the obvious variations in the samples (time, place, population, etc.) used in these studies, several other methodological reasons may also contribute to the cross-study variation summarized above. First, Hawcroft and Milfont (2010) demonstrate that both the length of the NEP scale $(6,12$, or 15 items) and the choices of items (in studies not using the full scales) can affect the findings on the factor structure of the NEP. Second, many studies using one form of exploratory factor analysis (e.g., Brennan et al., 2014; Englis \& Phillips, 2013; Ji, 2004; Noe \& Snow, 1990; Woodworth et al., 2011; Wu, 2012) tend to find factors that mostly reflect sample-specific methodological artifacts, such as survey question wording direction (see a review of this issue in Dunlap et al., 2000). Third, scholars also use different practices when interpreting results that can cause inconsistencies in their interpretation of findings. Some results on model-fit or interfactor correlations that were judged as supporting the multidimensional claim in the past (e.g., Geller \& Lasley, 1985; Nooney et al., 2003) could actually be interpreted differently depending on the criteria used. In fact, Dunlap (2008) argues that some measures of unidimensionality may be unrealistic. 
In sum, the current literature on the dimensionality of the NEP scale, although considerable, is largely inconclusive, as it was prior to 2000 (Dunlap et al., 2000). Much of the attention on dimensionality focuses on methodological issues, while implicitly assuming there is a coherent ecological world view that can be observed at this point, and therefore the debate is whether the NEP scale is adequate to capture it. We argue that this implicit assumption itself needs further attention.

\section{Reconceptualizing the dimensionality of the NEP}

A high degree of coherence of the NEP scale is of crucial importance if we are to treat the NEP as an ecological world view (e.g., Dunlap \& Van Liere, 1978; Dunlap et al., 2000; Guber, 1996; Nooney et al., 2003; Pierce \& Lovrich, 1980). Switching the focus of attention to the structure and coherence of the NEP is essentially going back to the aforementioned question for which the NEP scale was originally designed: is there a coherent ecological world view that has emerged among the general public at this point? Indeed, this switch would then turn the debate on the dimensionality of the NEP from a simplistic dichotomous question-is the NEP uni- or multi-dimensional-into a much more dynamic discussion on the degree of coherence of the NEP across time, samples, and even cultures, as well as the variables that may influence the coherence of the NEP.

Of course, to enable such a discussion of the coherence of the NEP in a comparative approach using the NEP scale, one necessary condition is needed: that the NEP scale is a valid and reliable measure. For this assertion, the current literature provides ample support. First, as Dunlap and colleagues (Dunlap 2008; Dunlap \& Van Liere, 1978; Dunlap et al., 2000) illustrate, the construction of the NEP scale is theoretically guided (although not in the sense of attitude theory as Dunlap points out regretfully in his 2008 retrospective essay). The NEP scale primarily targets the main elements of the DSP, which includes (as Pirages \& Ehrlich, 1974, among others, point out) a belief in unlimited resources, a commitment to progress and continuous economic growth, faith in science and technology, and a strong endorsement of a laissez-faire economy and private property (also see Kilbourne \& Beckmann, 2001; Milbrath, 1984; Takács-Sánta, 2007). This helps to explain the choice of facets included in the 1978 version of the NEP scale and the later updated 2000 version.

Second, the validity and reliability of the NEP scale are well established in the empirical literature (e.g., Dunlap et al., 2000; Hawcroft \& Milfont, 2010). Even on the dimensionality front, we see that many studies find the NEP scale likely unidimensional within certain groups and populations (e.g., Bechtel et al., 1999; Edgell \& Nowell, 1989; Lee \& Paik, 2011; Noe \& Snow, 1990; Rideout, 2014; Rideout et al., 2005). Others report strong interfactor correlations, suggesting a high degree of internal consistency (e.g., Fleury-Bahi et al., 2015; Nooney et al., 2003). 
We therefore argue that it is reasonable to treat the NEP scale as a valid and reliable measure, and that we can reinterpret the many findings of multidimensionality as not showing the inadequacy of the NEP scale, but revealing the variation in the degree of coherence of the NEP within different groups and populations. Indeed, as a new social paradigm, it would be unrealistic to expect that the NEP be universally and consistently accepted in human societies shortly after it emerges. On the contrary, we might anticipate fluctuations in the degree of acceptance and hence the coherence of the NEP over time and across samples and cultures. In fact, as Dunlap (2008, p. 15) points out, the diffusion of an ecological world view has been slow, the proponents of the DSP have mounted powerful and effective opposition to the spread of the NEP, and "we are in the midst of a paradigm war, with two sides attempting to give highly divergent interpretations of ecological realities."

In the literature, several scholars similarly argue that an ecological world view may just be emerging, and still lacks widespread acceptance by the general public, often using the multidimensionality as supporting evidence (e.g., Brennan et al., 2014; Corral-Verdugo \& Armendariz, 2000; Erdogan, 2013; Khan et al., 2012; Wu, 2012). Some (e.g., Takács-Sánta, 2007, p. 31) argue for the importance of recognizing key barriers to sustained environmental concern, such as a faith in science and technology or a tendency of media sources to "defend the status quo"; while others (e.g., Lawrence \& Abrutyn, 2015) point to the slowly emerging knowledge of the interconnectedness of ecosystems, and the need for an expansion of what a society acknowledges as "local" environmental issues.

Thus, it is important to continue tracking changes in the ecological world view within different groups and populations. One promising approach is to take insights from the belief-systems perspective that has originated from political science. The belief-systems perspective developed by political scientist Philip Converse defines a belief system as "a configuration of ideas and attitudes in which the elements are bound together by some form of constraint or functional interdependence," where constraint means "the success we would have in predicting, given initial knowledge that an individual holds a specified attitude, that he holds certain further ideas and attitudes" (Converse 1964, p. 207). Constraint as so defined refers to the interconnectedness among different beliefs within the same presumed belief system. As such, the NEP can be seen as a belief system, and its coherence can be reconceptualized as "belief constraint" at the group level because it also concerns interrelations among different beliefs within a given group.

\section{The structure and coherence of the NEP as a belief system}

In the literature of environmental concern, Pierce and Lovrich (1980) were among the first to use the belief-system perspective and found in their empirical study of environmental belief systems that "beliefs about specific environmental issues seem to fit together on a single dimension, thus suggesting some underlying 
concept or fundamental orientation to which these beliefs are mutually connected" (Pierce \& Lovrich, 1980, p. 261). Later, Pierce et al. (1987, p. 64) define belief constraint as "the extent to which an individual's beliefs are bound together or integrated into a larger, single complex," consistent with the definition offered by Converse (1964). According to Pierce and colleagues, environmental concern as a belief system clearly reflects belief constraint. However, deHaven-Smith (1991) argues, based on empirical studies of one national and one Florida survey, that the general public's environmental belief system tends to be largely fragmented, localized, and issue-specific, or lacks belief constraint. In these studies, the debate on the dimensionality of environmental concern evolved into an examination of the existence of belief constraint within the environmental belief system.

A number of studies explicitly apply the belief-system perspective to examine the NEP. Pierce et al. (1989) examine the organization of an environmental belief system using a 6-item NEP scale derived from the original 1978 12-item NEP scale, with attention not on the dimensionality of the scale, but on the conceptual structure across different samples. Nooney et al. (2003, p. 765) interpret the persistent debate on the dimensionality as a reflection on the lack of agreement regarding whether the NEP is a unified system of beliefs, and therefore argue that attention is needed on the "magnitude and direction of covariance between latent factors" to gain insight about "the cultural organization of environmental world views." Woodworth et al. (2011), on the other hand, argue their analysis affirms an emerging consensus that the NEP measures a coherent set of environmental beliefs; or an environmental paradigm or world view.

Several studies (Bechtel et al., 2006; Bechtel et al., 1999) use the NEP scale as a measure of an environmental belief system and examine how culture may influence the structure of environmental beliefs in cross-national comparative research. Results from these studies show that, in general, the NEP in the United States tends to show a higher degree of coherence than elsewhere. Several others compare and contrast the factor structure of the NEP cross-nationally and find significant variations, but do not apply the belief-systems perspective explicitly (e.g., Brennan et al., 2014; Denis \& Pereira, 2014).

In sum, studies utilizing the belief-systems perspective show a general shift of attention from methodological concerns on the adequacy of the NEP scale to interesting questions on the existence of and changes in an environmental world view. Dunlap (2008) in his review of the 30-year history of the NEP scale specifically calls for additional studies of this kind, applying the belief-systems perspective so that we can document variation in both the structure and coherence of an environmental world view. In our current study, we answer to this call. 


\section{Analysis strategy}

In this study, we obtained data from two independent surveys, one in 1997 and the other in 2012, held in three communities in Colorado and Wyoming, United States (more on this later). Both surveys used identical questionnaires, providing a good opportunity to examine and compare the structure and coherence of the NEP between two time points within the same geographic areas. To accomplish this, we followed the literature to make use of a theory-driven confirmatory factor analysis (CFA). CFA is known to be superior to exploratory factor analysis, for it has the ability to incorporate and test a preset factor structure commonly proposed in the literature, and also to run statistical tests for significances while controlling potential sample-specific correlations among measurement errors (e.g., Geller \& Lasley, 1985; Guber, 1996; Nooney et al., 2003; Xiao \& Dunlap, 2007).

Because the first survey in this study took place in 1997, prior to the publication of the revised NEP scale, we examined the original NEP scale for the 2012 survey as well, for comparison purposes. According to Dunlap and Van Liere (1978), the original NEP scale was designed to capture three primary aspects of the new social paradigm: limits to growth (LG), balance of nature (BN), and anti-anthropocentrism (AA). We therefore modeled each as a latent factor in our analysis. Each latent factor has four survey items as measurement indicators (a total of 12 items). To examine whether these three latent factors form a coherent ecological world view as Dunlap and Van Liere (1978) argue, we modeled one higher-order latent factor named "NEP" as measured by these three first-order latent factors, effectively forming a higher-order CFA as described in Amburgey and Thoman (2012). We fit this model to the data of both surveys simultaneously, using a multigroup modeling technique to increase estimation efficiency (see Byrne, 2012, for more technical details). Table 1 displays these 12 items and their corresponding latent factors and Figure 1 shows the path diagram of our CFA model.

Table 1. Twelve items of the new environmental paradigm scale and their corresponding factors

\begin{tabular}{|c|c|c|}
\hline Variable & Wording $^{\star \star}$ & Factor \\
\hline NEP1 & $\begin{array}{l}\text { We are approaching the limit of the number of people that the } \\
\text { Earth can support. }\end{array}$ & Limits to growth \\
\hline NEP2 & The balance of nature is very delicate and easily upset. & Balance of nature \\
\hline NEP3* & $\begin{array}{l}\text { Humans have the right to modify the natural environment to } \\
\text { suit their own needs. }\end{array}$ & Anti-anthropocentrism \\
\hline NEP4* & Humankind was created to rule over the rest of nature. & Anti-anthropocentrism \\
\hline NEP5 & $\begin{array}{l}\text { When humans interfere with nature, it often produces } \\
\text { disastrous consequences. }\end{array}$ & Balance of nature \\
\hline NEP6* & Plants and animals exist primarily to be used by humans. & Anti-anthropocentrism \\
\hline NEP7 & $\begin{array}{l}\text { To maintain a healthy environment, we will have to develop a } \\
\text { "steady state" economy where industrial growth is controlled. }\end{array}$ & Limits to growth \\
\hline
\end{tabular}


Human Ecology Review, Volume 23, Number 1, 2017

\begin{tabular}{|l|l|l|}
\hline Variable & Wording $^{* *}$ & Factor \\
\hline NEP8 & Humans must live in harmony with nature in order to survive. & Balance of nature \\
\hline NEP9 & $\begin{array}{l}\text { The Earth is like a spaceship with only limited room and } \\
\text { resources. }\end{array}$ & Limits to growth \\
\hline NEP10* & $\begin{array}{l}\text { Humans don't need to adapt to the natural environment, } \\
\text { because they can remake it to suit their needs. }\end{array}$ & Anti-anthropocentrism \\
\hline NEP11 & $\begin{array}{l}\text { There are limits to growth beyond which our industrialized } \\
\text { society cannot expand. }\end{array}$ & Limits to growth \\
\hline NEP12 & Humankind is severely abusing the environment. & Balance of nature \\
\hline
\end{tabular}

*These four items are worded in an anti-NEP direction. Reverse coding is performed on them in our confirmatory factor analysis. ${ }^{*}$ Respondents were asked to indicate whether they agree or disagree with the listed statements, using a Likert scale (strongly agree, agree, neutral, disagree, strongly disagree).

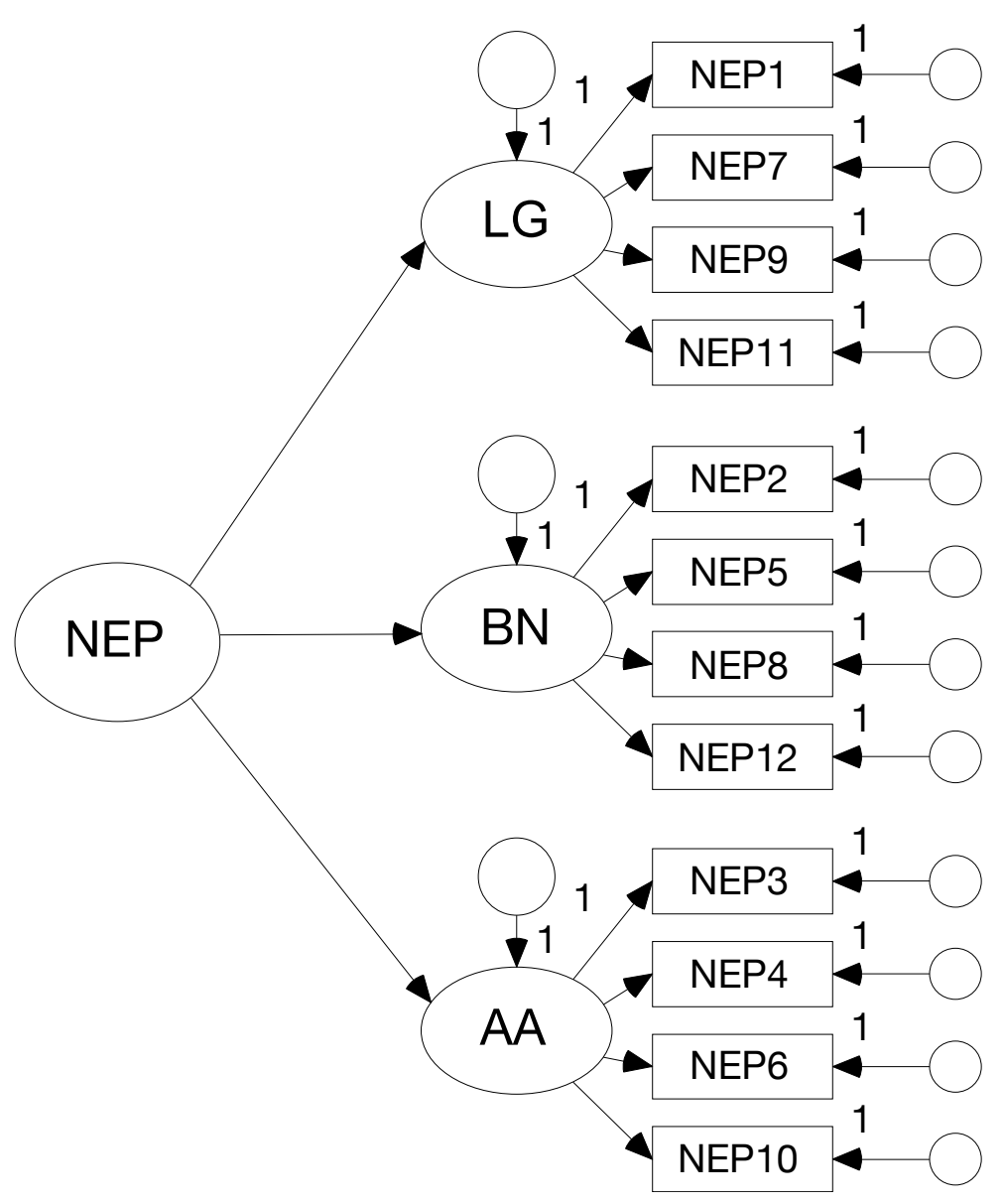

Figure 1. Path diagram of a higher-order confirmatory factor analysis examining the structure and coherence of the new environmental paradigm scale

Note. See Table 1 for details of all latent and observed variables. 


\section{Methodology}

\section{Samples, data, and study areas}

The 1997 survey used in this study is based on initial work by Nielsen and Ellington (1983), and includes the original NEP scale items, as well as additional questions on recycling and other environmentally conscious behaviors, attitudes on environmental policies, and demographics. The samples used in this survey were developed in 1997 and included three communities in Colorado and Wyoming, United States. An initial random sample of 200 households was collected for one community (Park Hill, part of the greater Denver, Colorado, area). Two additional random samples of 200 households were developed for Loveland, Colorado and Cheyenne, Wyoming. These three communities were chosen specifically because of the different levels of recycling services available to residents in 1997.

Loveland is a city of just over 70,000 in 2012, increasing from 46,000 in 1997, located approximately 50 miles north of Denver and approximately 60 miles southwest of Cheyenne. Politically, the City of Loveland is a "mixed" community of more politically conservative and more liberal residents. According to the US Census in 2010, 38\% of voters were registered as Republican, 37\% of registered voters were non-partisan, and $23 \%$ were registered as Democrats. This community is primarily Caucasian/white (91\% in 2010, down just slightly from 93\% in 2000).

The community of Park Hill is part of the larger Denver metropolitan area, and had a population in 2010 of just over 29,700 (up from 27,000 in 1997). While political party information is not available for the Park Hill neighborhood specifically, the larger Denver area tends to be more heavily Democratic (48\% of registered voters in 2010), while non-partisan voters made up 35\%, and Republicans made up 15\% of registered voters in 2010. In 1990, this community had a larger percentage of African-American residents (60\%), as compared to whites/Caucasians (34\%) (Park Hill Neighborhood Plan, 2000, p. 100).

The City of Cheyenne, Wyoming, had a population of 61,600 in 2012, up from 54,000 in 1997 . This community is primarily white/Caucasian ( $87 \%$ in 2010 and $88 \%$ in 2000), and included a majority of Republican voters (60 per cent), as compared to those registered Democratic (28\%) and non-partisan (11 per cent).

The mean rate of return for the 1997 study was 60\% (Loveland 62\%, Cheyenne 59\%, and Denver 60\%). For the 2012 survey, we used identical addresses for each of the three communities. Where identical addresses were not available, addresses were substituted (by a random selection process) from identical census blocks, with updated names provided by the Public Works departments for the cities of Loveland

2 Demographic data cited in this section is from the U.S. Census: censusviewer.com 
and Cheyenne, and by the Greater Park Hill Neighborhood Association for the Park Hill community. The mean rate of return for the 2012 study was $63.7 \%$ - slightly higher than for the 1997 survey (Loveland 62\%, Cheyenne 64\%, and Denver 65\%).

Over the 15-year time span, it is likely that a majority of surveys were not completed by the same individuals who participated in the 1997 study. Core demographics for each of the three communities varied somewhat between the two survey years. Using multiple analysis of variance tests, we found statistically significant differences between the 1997 and 2012 samples $(p<.05)$ for both age and education. The 2012 sample included a greater percentage of individuals in the $65+$ age category, as well as a greater number of individuals holding college degrees. Similarly, we found that gender and income demographics were significantly different between the two years. The 2012 sample included a higher percentages of females and those with more than $\$ 60,000$ annual household income as compared to respondents in 1997 (see Table 2 for more details). Although comparisons of the demographic differences between these three communities is beyond the scope of the current study, an advantage we find in using these samples is that the study populations are not overly homogenous, allowing for a greater degree of generalizability to larger populations. However, we must note that this is a rather small sample from just a few communities in two states of the United States, thus our ability to make generalizations to broader areas is still limited.

Table 2. Descriptive statistics of the samples in $1997(N=364)$ and 2012 $(N=382)$

\begin{tabular}{|c|c|c|c|c|c|c|}
\hline \multirow{2}{*}{\begin{tabular}{|l|} 
Variables \\
Age $^{\star}$ \\
\end{tabular}} & \multirow[t]{2}{*}{ Year } & \multicolumn{5}{|c|}{ Coding } \\
\hline & & $18-25$ & $26-40$ & $41-65$ & $65+$ & \\
\hline & 1997 & $2.92 \%$ & $28.90 \%$ & $48.38 \%$ & $19.81 \%$ & \\
\hline & 2012 & $1.36 \%$ & $16.08 \%$ & $42.51 \%$ & $40.05 \%$ & \\
\hline \multicolumn{2}{|l|}{ Income $^{*}$} & Under $\$ 30 \mathrm{k}$ & $\$ 30-60 k$ & Over $\$ 60 \mathrm{k}$ & & \\
\hline & 1997 & $29.06 \%$ & $35.31 \%$ & $35.63 \%$ & & \\
\hline & 2012 & $16.96 \%$ & $32.14 \%$ & $50.89 \%$ & & \\
\hline \multicolumn{2}{|l|}{ Education* } & $<$ High school & High school & Some college & College & Graduate degree \\
\hline & 1997 & $4.67 \%$ & $13.74 \%$ & $30 \%$ & $25 \%$ & $21.15 \%$ \\
\hline & 2012 & $1.05 \%$ & $13.61 \%$ & $28.01 \%$ & $25.13 \%$ & $27.23 \%$ \\
\hline \multicolumn{2}{|l|}{ Gender* } & Male & Female & & & \\
\hline & 1997 & $43.13 \%$ & $50.27 \%$ & & & \\
\hline & 2012 & $38.74 \%$ & $55.24 \%$ & & & \\
\hline
\end{tabular}

*Statistically significant difference found between 1997 and 2012 ( $p<$.05).

Dunlap (2008) notes that the United States culture is in the midst of a paradigm shift, gradually moving away from the tenets of the dominant social paradigm toward a belief system that puts a greater level of priority on environmental quality and protection. However, he also notes that this new paradigm has come under significant attack and 
criticism for the last few decades by those whose interests would be negatively affected by substantial shifts away from fossil fuels and many other aspects of consumer culture. For this reason, it is important to measure the degree of belief constraint over time (in the case of the current study, a 15-year period) to assess the extent to which environmental beliefs and concern have held steady or been influenced by those who continue to call into question the reality of climate change, or the need to find both short- and long-term alternatives to fossil fuels, for example.

Table 3 reports frequency distributions for the 12 NEP items for the two years. We tested differences in these 12 items between the two years and found none that were statistically significant.

Table 3. Frequency distribution (\%) of the new environmental paradigm items in $1997(N=364)$ and $2012(N=382)$

\begin{tabular}{|l|c|c|c|c|c|c|}
\hline Variable* & Year & Strongly Agree & Agree & Neutral & Disagree & Strongly Disagree \\
\hline NEP1 & 1997 & 16.67 & 33.33 & 22.50 & 16.11 & 6.11 \\
\hline & 2012 & 18.32 & 32.72 & 22.77 & 15.45 & 6.02 \\
\hline NEP2 & 1997 & 31.67 & 39.17 & 13.33 & 10 & 2.50 \\
\hline & 2012 & 30.37 & 37.70 & 17.28 & 7.33 & 3.40 \\
\hline NEP3 & 1997 & 1.67 & 11.67 & 19.72 & 41.67 & 21.39 \\
\hline & 2012 & 3.40 & 11.78 & 20.68 & 44.76 & 15.18 \\
\hline NEP4 & 1997 & 8.06 & 11.39 & 17.50 & 29.17 & 29.17 \\
\hline & 2012 & 7.33 & 10.21 & 14.14 & 31.15 & 32.46 \\
\hline NEP5 & 1997 & 24.17 & 45.56 & 13.06 & 8.33 & 4.44 \\
\hline & 2012 & 22.77 & 39.79 & 19.90 & 8.90 & 4.45 \\
\hline NEP6 & 1997 & 7.50 & 11.11 & 17.78 & 36.67 & 21.94 \\
\hline & 2012 & 4.71 & 10.21 & 17.54 & 37.43 & 25.65 \\
\hline NEP7 & 1997 & 13.89 & 34.17 & 31.11 & 10.28 & 5.28 \\
\hline & 2012 & 12.83 & 31.41 & 32.46 & 11.26 & 5.76 \\
\hline NEP8 & 1997 & 42.22 & 43.06 & 6.94 & 1.39 & 1.67 \\
\hline & 2012 & 39.79 & 41.36 & 8.38 & 3.40 & 2.88 \\
\hline NEP9 & 1997 & 30.83 & 38.33 & 13.89 & 8.89 & 2.50 \\
\hline & 2012 & 24.87 & 37.70 & 17.02 & 10.99 & 3.93 \\
\hline NEP10 & 1997 & 1.94 & 6.94 & 10.56 & 39.44 & 34.72 \\
\hline & 2012 & 1.05 & 3.40 & 13.09 & 43.72 & 33.77 \\
\hline NEP11 & 1997 & 20.83 & 40.28 & 22.50 & 6.94 & 2.22 \\
\hline & 2012 & 14.92 & 36.39 & 27.49 & 9.69 & 4.19 \\
\hline NEP12 & 1997 & 31.94 & 40.00 & 13.06 & 7.50 & 3.06 \\
\hline & 2012 & 30.37 & 34.82 & 18.32 & 6.54 & 5.24 \\
\hline
\end{tabular}

Note. No statistically significant difference found between 1997 and 2012 in any comparison.

*For descriptions of each variable, see Table 1. 
We examined patterns of missing data before we estimated our confirmatory factor analysis model. We found that 28 cases had missing values on at least $10 \mathrm{NEP}$ items and hence were deleted from the data set. For the remaining missing data, we chose to impute them using an imputation tool included in the statistical software Mplus 7.11. To account for random errors that may occur during this imputation process, particularly considering the relatively small sample size, we followed Rubin (1987) to obtain five imputed data sets, which we then analyzed to provide five sets of model results. Our final results were acquired by properly averaging results from these five sets of results (see Rubin, 1987, for more technical details).

With missing data dealt with, we proceeded to model estimation using Mplus 7.11. Table 4 reports relevant model estimation results. Mplus provides multiple indexes to gauge how well our model fits the sample data (reported in Table 4). These model-fit indexes generally show this model has acceptable to good fit, with $>0.95$ comparative fit index and Tucker-Lewis index, as well as a reasonably small root mean square error of approximation $($ RMSEA $=0.07)$.

Table 4. Results of a higher-order confirmatory factor analysis examining the structure and coherence of the new environmental paradigm scale

\begin{tabular}{|c|c|c|c|}
\hline \multirow[b]{2}{*}{ First-order factors } & \multirow[b]{2}{*}{ Item } & \multicolumn{2}{|c|}{ Standardized loadings (standard error) } \\
\hline & & $1997(N=351)$ & $2012(N=367)$ \\
\hline \multirow[t]{4}{*}{ LG (limits to growth) } & NEP1 & $0.60(0.04)$ & $0.60(0.04)$ \\
\hline & NEP7 & $0.64(0.04)$ & $0.70(0.03)$ \\
\hline & NEP9 & $0.73(0.04)$ & $0.71(0.04)$ \\
\hline & NEP11 & $0.70(0.04)$ & $0.70(0.03)$ \\
\hline \multirow[t]{4}{*}{ BN (balance of nature) } & NEP2 & $0.77(0.03)$ & $0.84(0.02)$ \\
\hline & NEP5 & $0.71(0.03)$ & $0.68(0.03)$ \\
\hline & NEP8 & $0.77(0.03)$ & $0.73(0.03)$ \\
\hline & NEP12 & $0.81(0.03)$ & $0.83(0.02)$ \\
\hline \multirow[t]{4}{*}{ AA (anti-anthropocentrism) } & NEP3 & $0.73(0.04)$ & $0.70(0.03)$ \\
\hline & NEP4 & $0.74(0.04)$ & $0.79(0.03)$ \\
\hline & NEP6 & $0.62(0.05)$ & $0.70(0.04)$ \\
\hline & NEP10 & $0.67(0.04)$ & $0.75(0.04)$ \\
\hline \multicolumn{2}{|l|}{ Second-order factor } & \multicolumn{2}{|c|}{ Standardized loadings (standard error) } \\
\hline \multirow[t]{3}{*}{ NEP } & $L G^{*}$ & $0.85(0.03)$ & $0.96(0.03)$ \\
\hline & BN & $0.99(0.03)$ & $0.95(0.02)$ \\
\hline & AA & $0.74(0.05)$ & $0.74(0.04)$ \\
\hline \multicolumn{2}{|l|}{ Interfactor correlations } & \multicolumn{2}{|c|}{ Correlations (standard error) } \\
\hline \multicolumn{2}{|l|}{$\mathrm{LG}$ and $\mathrm{BN}$} & $0.84(0.03)$ & $0.91(0.02)$ \\
\hline \multicolumn{2}{|l|}{ LG and $A A$} & $0.63(0.05)$ & $0.71(0.04)$ \\
\hline \multicolumn{2}{|l|}{$\mathrm{BN}$ and $\mathrm{AA}$} & $0.73(0.04)$ & $0.70(0.04)$ \\
\hline
\end{tabular}


The Structure and Coherence of the New Environmental Paradigm

\begin{tabular}{|l|c|}
\hline Model-fit statistics \\
\hline Chi-square/degree of freedom & $313.06 / 113$ \\
\hline RMSEA (root mean square error of approximation) & 0.07 \\
\hline CFI (comparative fit index) & 0.97 \\
\hline TLI (Tucker-Lewis fit index) & 0.97 \\
\hline
\end{tabular}

Note. All factor loadings and correlations are statistically significant $(p<.05)$. Interfactor correlations are estimated using an alternative model that has no second-order latent factor but only three correlated first-order latent factors.

*Statistically significant difference found between the two loadings $(p<.01)$.

\section{Results and discussions}

We begin with a discussion of results related to the structure of the NEP scale, which examines the conceptual integrity of the NEP. First, each NEP item has a strong factor loading $(0.60-0.84)$ on its hypothesized latent factor in both years. Second, each first-order latent factor has a very strong (0.85-0.99) loading on the second-order latent factor, the NEP. Thus, our model confirms the overall factor structure as proposed in Dunlap and Van Liere (1978), that the NEP scale has three facets as conceptually designed-limits to growth, balance of nature, and anti-anthropocentrism - which all then load onto the same overarching world view named the NEP.

It is important to note that many second-order loadings are significantly less than 1.00. Indeed, a Wald test of model-fit differences between this current model and an alternative model with all 12 items loading onto one single latent factor shows statistical significance (Chi-square $=52.67$, degree of freedom $=6, p<.05$ ), indicating that our current trifactor model fits the data significantly better than the one-factor model. Thus, this current model fails to confirm a unidimensional factor structure. However, the very strong second-order factor loadings show that the three first-order latent factors have very high covariances between them. We used an alternative model that has no second-order latent factor but only three correlated first-order latent factors to obtain estimations of interfactor correlations (also reported in Table 4). Results show that the three latent factors (LG, BN, and AA) are highly correlated with one another. The correlation between LG and BN is especially high in both years, while AA is a relatively distinct facet, as is often found in the literature (Dunlap et al., 2000). Therefore, we conclude that the NEP scale has a multidimensional factor structure with three strongly correlated underlying factors.

We now turn our attention to the coherence of the NEP. As discussed above, coherence can be conceptualized as belief constraint at the group level that refers to the interconnectedness among different beliefs within the belief system. Thus, 
coherence is a matter of degree. In the framework of factor analysis, factor loadings display how closely survey items connect to the hypothesized underlying concepts. Therefore, factor loadings can illustrate the degree of interconnectedness. However, one problem is that there is no widely agreed objective standard, or a cutoff value, that we can use to judge the interconnectedness, making the evaluation of factor loading magnitude ultimately arbitrary.

In the literature of factor analysis, a number of cutoff values of factor loading are used, ranging from 0.20 to $0.70 ; 0.40$ is the most commonly used, 0.30 the second, and 0.50 the third (Peterson, 2000). Our results show that even the lowest of all first-order factor loadings, 0.60 to be exact, is above the more restrictive common cutoff of 0.50 . For the 1997 survey, all 12 first-order factor loadings have a range of $0.60-0.81$ and a mean of 0.71 ; for 2012 the range is $0.60-0.84$ and the mean 0.73 . Therefore, it is reasonable to conclude that all three facets (LG, BN, and AA) have a good degree of coherence within each individual facet. Furthermore, all three facets strongly load onto the second-order latent factor, the NEP, with all loadings above even the highest cutoff of 0.70 . For 1997, the second-order factor loadings have a range of $0.74-0.99$ and a mean of 0.86 ; for 2012 the range is $0.74-0.96$ and the mean 0.88 . Thus, we conclude the NEP within the two samples of our study has a high degree of coherence or belief constraint.

Next, we compare results of the two years. Our first step is to compare the overall factor structure between the years, which examines how stable the conceptual structure of NEP has been over the years. As already mentioned, our analysis suggests the same factor structure exists in both years, essentially confirming that the NEP has three correlated facets as hypothesized (Table 4). In the second step, we compare, one by one, each of the 12 first-order factor loadings. These factor loadings are remarkably consistent between the two years. In fact, none of the 12 tests for factor loading differences is statistically significant.

Lastly, we compare the three pairs of second-order factorings. Once again, these loadings are quite consistent between the two surveys, with only one statistically significant result showing that limits to growth loads significantly higher on the NEP in 2012 than $1997(p<.001)$. However, since both loadings are already very high at 0.96 and 0.85 , respectively, this difference is of little substantive meaning. With these comparisons, we conclude that the NEP has the same factor structure and degree of coherence in both years of 1997 and 2012.

Because the above comparison also largely establishes measurement invariance between the two independent samples (Byrne, 2012), we took the opportunity to conduct tests for differences in latent means, first of the three facets, then of the overall NEP, between the years. No results were statistically significant, indicating no significant change found in the levels of endorsement of the NEP between 1997 and 2012. With this finding of the same levels of endorsement, and also the findings 
of the same factor structure and degree of coherence in the years of 1997 and 2012, we conclude that the NEP has remained conceptually stable between the two survey years, even though the two independent samples are significantly different from one another in terms of age, household income, education, and percentages of males and females.

With the above results, we now have a similar situation to that of several previous studies (e.g., Amburgey \& Thoman, 2012; Nooney et al., 2003), where the NEP is found to be multidimensional, but the underlying factors are closely correlated with one another. How then, should we draw conclusions about the coherence of the NEP, enough to support the existence of an environmental world view? We believe that despite the finding of multidimensionality, there is ample support for a coherent environmental world view that also exhibits remarkable stability within the general public, at least in the study areas of our two surveys.

As previously mentioned, we believe that some criteria for unidimensionality and hence the existence of a coherent world view is unrealistic. The NEP scale, both the original and revised versions, is designed to capture a set of different but connected beliefs or views regarding the relationship between human society and the environment. In the framework of confirmatory factor analysis, this means a model with multiple latent factors. A unidimensional model means one single latent factor, either at the first order with all measurement items loading onto it, or at the second order with each first-order latent factor loading onto it perfectly. Thus, to achieve unidimensionality, one would need to find no statistically significant difference in the model-fit comparison between these two models, effectively testing against the null hypothesis that all first-order latent factors have either interfactor correlations or standardized factor loadings at 1.00. Such a null hypothesis is unreasonably strong and restrictive, not only because a magnitude of 1.00 for correlation or factor loading is typically unattainable in real-world research, but also that is somewhat unfair, since in exploratory factor analysis we almost never expect the loadings of survey items to approach 1.00 (e.g., Peterson, 2000). We thus argue that multidimensionality does not necessarily deny the existence of a coherent belief system without a careful examination of the coherence indicators, such as secondorder factor loadings and interfactor correlations.

\section{Conclusion}

In this study, we used data from two independent surveys of three communities in Colorado and Wyoming in 1997 and 2012 to examine the structure and coherence of an environmental world view as measured by the NEP scale. We propose that the debate on the dimensionality of the NEP scale be reconceptualized as variation in the factor structure and degree of coherence of the NEP, or, in the terminology of 
belief-systems perspective, the degree of belief constraint. Our results show that, similar to several previous studies (e.g., Amburgey \& Thoman, 2012; Nooney et al., 2003), the NEP in our study area is likely multidimensional with three facets-limits to growth, balance of nature, and anti-anthropocentrism-but these three facets are highly correlated with one another, indicating a high degree of coherence or belief constraint. Comparisons between the two years reveal that both the structure and coherence of the NEP are highly consistent over time, displaying remarkable stability. With such findings, we believe that the general public within our study areas indeed has a coherent environmental world view.

Next, we offer several insights for future research, reflecting upon the limitations of the current study. First, our study employs the original version of the new environmental paradigm scale, not the updated new ecological paradigm scale. We believe future studies should reexamine the stability and coherence of the NEP using this newer version of the NEP scale with a longitudinal design. Second, because this study has its origin in research for a doctoral dissertation, it uses small samples that are limited to a few local communities in only two states within the United States. It will be important to expand the research onto the national and international levels. Finally, the finding of the remarkable stability of the NEP also deprives us of the chance to look into additional factors that may influence the structure and belief constraint. As we mentioned earlier, there are reasons to be cautious about the extent to which the general public may have adopted this new social paradigm/ world view. For this reason, it is crucial to anticipate variation in the coherence of the NEP and study its causes. Several cross-national studies (e.g., Bechtel et al., 2006; Bechtel et al., 1999) use a comparative research design to demonstrate that cultural differences may influence the structure and belief constraint of the NEP. Similarly, Aoyagi-Usui et al. (2003) and Heyd (2010) highlight the importance of examining cultural norms and traditions in terms of their impact on environmental values and beliefs. We thus call for more such research in the future.

\section{References}

Albrecht, D., Bultena, G., Holberg, E., \& Nowak, P. (1982). The new environmental paradigm scale. Journal of Environmental Education, 13(3), 39-43. doi.org/10.1 080/00958964.1982.9942647

Amburgey, J. W., \& Thoman, D. B. (2012). Dimensionality of the new ecological paradigm: Issues of factor structure and measurement. Environment and Behavior, 44(2), 235-256. doi.org/10.1177/0013916511402064

Aoyagi-Usui, M., Vinken, H., \& Kuribayashi, A. (2003). Pro-environmental attitudes and behaviors: An international comparison. Human Ecology Review, 10(1), 23-31. 
Bechtel, R. B., Corral-Verdugo, V., Asai, M., \& Gonzalez Riesle, A. (2006). A cross-cultural study of environmental belief structures in USA, Japan, Mexico, and Peru. International Journal of Psychology, 41(2), 145-151. doi.org/ $10.1080 / 00207590500345401$

Bechtel, R. B., Corral-Verdugo, V., \& Pinheiro, J. (1999). Environmental belief systems: United States, Brazil, and Mexico. Journal of Cross-Cultural Psychology, 30(1), 122-128. doi.org/10.1177/0022022199030001008

Brennan, L., Binney, W., Aleti, T., \& Parker, L. (2014). Why validation is important: An example using the NEP scales. Market and Social Research, 22(2), 15-31.

Byrne, B. (2012). Structural equation modeling with Mplus. New York, NY: Routledge.

Converse, P. E. (1964). The nature of belief system in mass public. In D. E. Apter (Ed.), Ideology and discontent (pp. 206-261). New York, NY: The Free Press of Glencoe.

Corral-Verdugo, V., \& Armendariz, L. I. (2000). The 'new environmental paradigm' in a Mexican community. Journal of Environmental Education, 31(3), 25-31. doi.org/10.1080/00958960009598642

deHaven-Smith, L. (1991). Environmental concern in Florida and the nation. Gainesville, FL: University of Florida Press.

Denis, H. D., \& Pereira, L. N. (2014). Measuring the level of endorsement of the new environmental paradigm: A transnational study. Dos Algarves: A Multidisciplinary e-Journal, 23, 4-26.

Dunlap, R. E. (2008). The new environmental paradigm scale: From marginality to worldwide use. Journal of Environmental Education, 40(1), 3-18. doi.org/ 10.3200/JOEE.40.1.3-18

Dunlap, R. E., \& Van Liere, K. (1978). The new environmental paradigm. Journal of Environmental Education, 9(4), 10-19. doi.org/10.1080/00958964. 1978.10801875

Dunlap, R. E., Van Liere, K. D., Mertig, A. G., \& Jones, R. E. (2000). Measuring endorsement of the new ecological paradigm: A revised NEP scale. Journal of Social Issues, 56(3), 425-442. doi.org/10.1111/0022-4537.00176

Edgell, M. C. R., \& Nowell, D. E. (1989). The new environmental paradigm scale: Wildlife and environmental beliefs in British Columbia. Society and Natural Resources, 2, 285-296. doi.org/10.1080/08941928909380692 
Englis, B. G., \& Phillips, D. M. (2013). Does innovativeness drive environmentally conscious consumer behavior? Psychology and Marketing, 30(2), 160-172. doi.org/10.1002/mar.20595

Erdogan, N. (2013). Exploring the new ecological paradigm scale on environmental worldviews of Turkish university students. AWERProcedia Advances in Applied Sciences, 1, 77-83.

Fleury-Bahi, G., Marcouyeux, A., Renard, E., \& Roussiau, N. (2015). Factorial structure of the new ecological paradigm scale in two French samples. Environmental Education Research, 21(6), 821-831. doi.org/10.1080/1350462 2.2014.913127

Furman, A. (1998). A note on environmental concern in a developing country: Results from an Istanbul survey. Environment and Behavior, 30(4), 520-534. doi.org/10.1177/001391659803000406

Geller, J. M., \& Lasley, P. (1985). The new environmental paradigm scale: A reexamination. Journal of Environmental Education, 17(1), 9-12. doi.org/10.1 080/00958964.1985.9941393

Gooch, G. D. (1995). Environmental beliefs and attitudes in Sweden and the Baltic states. Environment and Behavior, 27(4), 513-539. doi.org/10.1177/ 0013916595274004

Guber, D. L. (1996). Environmental concern and the dimensionality problem: A new approach to an old predicament. Social Science Quarterly, 77(3), 643-662.

Hawcroft, L. J., \& Milfont, T. (2010). The use (and abuse) of the new environmental paradigm scale over the last 30 years: A meta-analysis. Journal of Environmental Psychology, 30, 143-158. doi.org/10.1016/j.jenvp.2009.10.003

Heyd, T. (2010). Climate change, individual responsibilities and cultural frameworks. Human Ecology Review, 17(2), 86-95.

Ji, C.-H. C. (2004). Factor structure of the new environmental paradigm scale: Evidence from an urban sample in southern California. Psychological Reports, 94, 125-130. doi.org/10.2466/pr0.94.1.125-130

Khan, A., Khan, M. N., \& Adil, M. (2012). Refinement and validation of new environmental paradigm (NEP) in India: A CFA approach. Paradigm, 16(1), 39-50. doi.org/10.1177/0971890720120106

Kilbourne, W. E., \& Beckmann, S. C. (2001). Rationality and the reconciliation of the DSP with the NEP. In S. C. Beckmann \& E. K. Madsen (Eds.), Environmental regulation and rationality: Multidisciplinary perspectives. Aarhus, Denmark: Aarhus University Press. 
Lawrence, K. S., \& Abrutyn, S. B. (2015). The degradation of nature and the growth of environmental concern: Toward a theory of the capture and limits of ecological value. Human Ecology Review, 21(1), 87-108.

Lee, S., \& Paik, H. S. (2011). Korean household waste management and recycling behavior. Building and Environment, 46, 1159-1166. doi.org/10.1016/j. buildenv.2010.12.005

Milbrath, L. (1984). Environmentalists: Vanguard for a new society. Albany, NY: SUNY Press.

Nielsen, J. M., \& Ellington, B. L. (1983). Social processes and resource conservation: A case study in low technology recycling. Journal of Environmental Education, 19, 38-41.

Noe, F. P., \& Snow, R. (1990). The new environmental paradigm and further scale analysis. Journal of Environmental Education, 21, 20-26. doi.org/10.1080/0095 8964.1990.9941934

Nooney, J. G., Woodrum, E., Hoban, T. J., \& Clifford, W. B. (2003). Environmental worldview and behavior: Consequences of dimensionality in a survey of North Carolinians. Environment and Behavior, 35(6), 763-783. doi.org/10.1177/0013916503256246

Park Hill Neighborhood Plan. (2000). www.denvergov.org/content/dam/denvergov/ Portals/646/documents/planning/Plans/Park_Hill_Neighborhood_Plan.pdf

Peterson, R. A. (2000). A meta-analysis of variance accounted for and factor loadings in exploratory factor analysis. Marketing Letters, 11(3), 261-275. doi.org/10.1023/A:1008191211004

Pierce, J. C., \& Lovrich, N. P., Jr. (1980). Belief systems concerning the environment: The general public, attentive publics, and state legislators. Political Behavior, 2(3), 259-286. doi.org/10.1007/BF00990482

Pierce, J. C., Lovrich, N. P., Jr., Tsurutani, T., \& Abe, T. (1987). Culture, politics and mass publics: Traditional and modern supporters of the new environmental paradigm in Japan and the United States. The Journal of Politics, 49(1), 54-79. doi.org/10.2307/2131134

Pierce, J. C., Lovrich, N. P., Jr., Tsurutani, T., \& Abe, T. (1989). Public knowledge and environmental politics in Japan and the United States. Boulder, CO: Westview Press.

Pirages, D. C., \& Ehrlich, P. R. (1974). Ark II: Social response to environmental imperatives. San Francisco, CA: W. H. Freeman. 
Rideout, B. E. (2014). The liberal arts and environmental awareness: Exploring endorsement of an environmental worldview in college students. International Journal of Environmental and Science Education, 9, 59-76.

Rideout, B. E., Hushen, K., McGinty, D., Perkins, S., \&Tate, J. (2005). Endorsement of the new ecological paradigm in systematic and e-mail samples of college students. Journal of Environmental Education, 36(2), 15-23. doi.org/10.3200/ JOEE.36.2.15-23

Rubin, D. B. (1987). Multiple imputation for nonresponse in surveys. New York, NY: Wiley. doi.org/10.1002/9780470316696

Schuett, M. A., \& Ostergren, D. (2003). Environmental concern and involvement of individuals in selected voluntary associations. Journal of Environmental Education, 34(4), 30-38. doi.org/10.1080/00958960309603485

Takács-Sánta, A. (2007). Barriers to environmental concern. Human Ecology Review, 14(1), 26-38.

Van Riper, C. J., \& Kyle, G. T. (2014). Capturing multiple values of ecosystem services shaped by environmental worldviews: A spatial analysis. Journal of Environmental Management, 145, 374-384. doi.org/10.1016/j.jenvman.2014. 06.014

Woodworth, B. L., Steen-Adams, M., \& Mittal, P. (2011). Role of an environmental studies course on the formation of environmental worldviews: A case study of a core curriculum requirement using the NEP scale. Journal of Environmental Studies and Sciences, 1, 126-137. doi.org/10.1007/s13412-011-0013-4

Wu, L. (2012). Exploring the new ecological paradigm scale for gauging children's environmental attitudes in China. Journal of Environmental Education, 43(2), 107-120. doi.org/10.1080/00958964.2011.616554

Xiao, C., \& Dunlap, R. E. (2007). Validating a comprehensive model of environmental concern cross-nationally: A U.S.-Canadian comparison. Social Science Quarterly, 88(2), 471-493. doi.org/10.1111/j.1540-6237.2007.00467.x 
This text is taken from Human Ecology Review, Volume 23, Number 1, 2017, published 2017 by ANU Press, The Australian National University, Canberra, Australia.

dx.doi.org/10.22459/HER.23.01.2017.09 